PROCEEDINGS OF THE

AMERICAN MATHEMATICAL SOCIETY

Volume 135, Number 3, March 2007, Pages 649-657

S 0002-9939(06)08504-2

Article electronically published on August 31, 2006

\title{
POINTED HOPF ALGEBRAS OF FINITE COREPRESENTATION TYPE AND THEIR CLASSIFICATIONS
}

\author{
GONGXIANG LIU AND FANG LI
}

(Communicated by Martin Lorenz)

\begin{abstract}
Let $k$ be an algebraically closed field. The main goal of this paper is to classify the finite-dimensional pointed Hopf algebras over $k$ of finite corepresentation type. To do so, we give a necessary and sufficient condition for a basic Hopf algebra over $k$ to be of finite representation type firstly. Explicitly, we prove that a basic Hopf algebra over $k$ is of finite representation type if and only if it is Nakayama. By this conclusion, we classify all finite-dimensional pointed Hopf algebras over $k$ of finite corepresentation type.
\end{abstract}

\section{INTRODUCTION}

In this paper, let $k$ be an algebraically closed field and all spaces are $k$-spaces.

We say a coalgebra $C$ is of finite corepresentation type if $C^{*}$ is of finite representation type. It is known that a finite-dimensional coalgebra $C$ is pointed if and only if $C^{*}$ is a basic algebra. So, the classifications of finite-dimensional pointed Hopf algebras over $k$ of finite corepresentation type are equivalent to the classifications of finite-dimensional basic Hopf algebras over $k$ of finite representation type. In this paper, this equivalence will be used frequently.

In the representation theory of algebras, one remarkable conclusion, due to $\mathrm{P}$. Gabriel, states that for any basic algebra $A$ over $k$, there exists a unique quiver $\Gamma_{A}$ such that $k \Gamma_{A} / I \cong A$ as algebras, where $J^{N} \subseteq I \subseteq J^{2}(N \geq 2)$ and $J$ is the ideal generated by all arrows. An advantage for this conclusion is that we can transform the study of $A$-modules to that of representations of path algebra with relations (see [1]).

One of our goals is to characterize finite-dimensional basic Hopf algebras of finite representation type by quivers. As a special kind of basic algebras, there must be some additional restrictions on the corresponding quiver on a basic Hopf algebra. Fortunately, Green and Solberg proved that the corresponding quiver must be a so-called covering quiver (see [6]). This fact is important for us.

Received by the editors September 11, 2004 and, in revised form, September 10, 2005 and September 23, 2005.

2000 Mathematics Subject Classification. Primary 16G20, 16G30, 16W30.

This project was supported by the Program for New Century Excellent Talents in University (No.04-0522), the Natural Science Foundation of Zhejiang Province of China (No.102028) and partially by the Cultivation Fund of the Key Scientific and Technical Innovation Project, Ministry of Education of China (No.704004). 
At first, we give the definition of covering quivers. As a byproduct, we discuss the relationship between covering quivers and Hopf quivers which were defined in 3. It turns out that they are equivalent (see Proposition 2.1). Some results from [6], which are needed in this paper, are recalled in Section 2.

In Section 3, we will give the main result, that is, a finite-dimensional basic Hopf algebra over $k$ is of finite representation type if and only if it is Nakayama (Theorem 3.1). In the viewpoint of representation theory, Nakayama algebras are the best understood Artin algebras next to semisimple algebras and their Auslander-Reiten quivers are given explicitly (see [1]). Thus, the Gabriel quivers and the AuslanderReiten quivers of basic Hopf algebras over an algebraically closed field of finite representation type are explicitly known.

By a conclusion in [2, we will show that a finite-dimensional pointed Hopf algebra over $k$ is of finite corepresentation type if and only if it is comonomial (see 2]). The authors of 2 classified all comonomial Hopf algebras when the characteristic of $k$ is zero. In [7, the first author of this paper and Y. Ye have given a description of the structures of comonomial Hopf algebras when the characteristic of $k$ is not zero. By these conclusions, we give the classifications of pointed Hopf algebras of finite corepresentation type in Section 4.

\section{Preliminaries}

This section relies heavily on two beautiful papers [5], 6] and the book [1].

Quivers considered here are always finite. Given a quiver $\Gamma=\left(\Gamma_{0}, \Gamma_{1}\right)$ with $\Gamma_{0}$ the set of vertices and $\Gamma_{1}$ the set of arrows, denote by $k \Gamma$ the path algebra of $\Gamma$. For $\alpha \in \Gamma_{1}$, let $s(\alpha)$ and $t(\alpha)$ denote respectively the starting and the ending vertex of $\alpha$. An ideal $I$ of $k \Gamma$ is admissible if $J^{N} \subseteq I \subseteq J^{2}$ for some positive integer $N \geq 2$, where $J$ is the ideal generated by all arrows.

For any finite-dimensional algebra $\Lambda$, we denote the Jacobson radical of $\Lambda$ by $J_{\Lambda}$. An algebra $\Lambda$ is said to be basic if $\Lambda \cong \bigoplus_{i=1}^{n} P_{i}$ for some indecomposable projective $\Lambda$-modules $P_{i}$; then $P_{i} \nsucceq P_{j}$ for $i \neq j$. It is known that a basic algebra $\Lambda$ over an algebraically closed field $k$ is elementary, i.e. $\Lambda / J_{\Lambda} \cong k \times k \times \cdots \times k$. A remarkable conclusion in representation theory, due to P. Gabriel, states that, for any elementary algebra $\Lambda$, there exists a unique finite quiver $\Gamma$ and an admissible ideal $I$ of $k \Gamma$, such that $\Lambda \cong k \Gamma / I$ (see [1]).

Next, let us recall the definition of covering quivers (see [6]). Let $G$ be a finite group and let $W=\left(w_{1}, w_{2}, \ldots, w_{n}\right)$ be a sequence of elements of $G$. We say $W$ is a weight sequence if, for each $g \in G$, the sequences $W$ and $\left(g w_{1} g^{-1}, g w_{2} g^{-1}, \ldots\right.$, $\left.g w_{n} g^{-1}\right)$ are the same up to a permutation. In particular, $W$ is closed under conjugation. Define a quiver, denoted by $\Gamma_{G}(W)$, as follows. The vertices of $\Gamma_{G}(W)$ comprise the set $\left\{v_{g}\right\}_{g \in G}$, and the arrows are given by

$$
\left\{\left(a_{i}, g\right): v_{g^{-1}} \rightarrow v_{w_{i} g^{-1}} \mid i=1,2, \ldots, n, g \in G\right\} .
$$

We call this quiver the covering quiver (with respect to $W$ ).

Example 2.1. (1): Let $G=\langle g\rangle, g^{n}=1$ and $W=(g)$. Then the corresponding covering quiver (we call such a quiver a basic cycle of length $n$ ) is pictured as follows. 


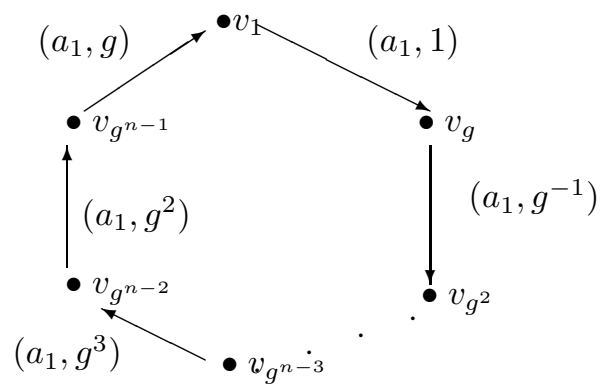

(2): Let $G=K_{4}=\{1, a, b, a b\}$, the Klein four group, and $W=(1)$. Then the corresponding covering quiver is

$$
\bullet^{1} \circlearrowleft, \bullet^{a} \circlearrowleft, \bullet^{b} \circlearrowleft, \bullet^{a b} \circlearrowleft
$$

At present, we digress to discuss the relationship between covering quivers and Hopf quivers, which are defined in 3 . Let us recall it.

Let $G$ be a finite group and $\mathcal{C}$ the set of conjugate classes. Denote the set of natural numbers by $\mathcal{N}$. A class function $\chi: \mathcal{C} \rightarrow \mathcal{N}$ is called a ramification, denoted by $\chi=\sum_{C \in \mathcal{C}} \chi_{C} C$. Given a ramification $\chi=\sum_{C \in \mathcal{C}} \chi_{C} C$ of $G$, then the corresponding Hopf quiver $\Gamma(G, \chi)$ has the set of vertices $\Gamma_{0}=G$, and for each $x \in \Gamma_{0}, c \in C \in \mathcal{C}$, one has $\chi_{C}$ arrows from $x$ to $c x$.

Given a covering quiver $\Gamma_{G}(W)$, where $W=\left(w_{1}, w_{2}, \ldots, w_{n}\right)$ is a weight sequence, since $W$ is closed under conjugation, $W$, as a set, equals the disjoint union of elements in some conjugate classes. Without loss of generality, assume $W$ is the disjoint union of elements in $C_{1}, C_{2}, \ldots, C_{m}$. Define a ramification $\chi$ by $\chi_{C}=$ multiplicity of $C$ in $\left\{C_{1}, C_{2}, \ldots, C_{m}\right\}$. Then, we can get that $\Gamma_{G}(W) \cong \Gamma(G, \chi)$ as directed graphs.

Conversely, let $\Gamma(G, \chi)$ be a Hopf quiver with $\chi=\sum_{C \in \mathcal{C}} \chi_{C} C$. Define $W$ to be the disjoint union of elements in $\chi_{C}$ copies of $C$. Since $W$ is a finite set, we can give an order on $W$ such that $W$ is a sequence. Clearly, $W$ is a weight sequence, $\Gamma_{G}(W)$ is a covering quiver and $\Gamma(G, \chi) \cong \Gamma_{G}(W)$ as directed graphs.

Combining these remarks, we get the following consequence.

Proposition 2.1. A quiver is a covering quiver if and only if it is a Hopf quiver.

The following conclusion (see Theorem 2.3 in [6]) states the importance of covering quivers.

Lemma 2.2. Let $H$ be a finite-dimensional basic Hopf algebra over $k$. Then there exists a finite group $G$ and a weight sequence $W=\left(w_{1}, w_{2}, \ldots, w_{n}\right)$ of $G$, such that $H \cong k \Gamma_{G}(W) / I$ for an admissible ideal $I$.

Next, let us recall a wonderful E. Green's conclusion, which plays a crucial role in the proof of the main theorem (Theorem 3.1).

There is a natural left $G$-action on $\Gamma_{G}(W)$. That is, $g \cdot v_{h}=v_{h g^{-1}}$ and $g$. $\left(a_{i}, h\right)=\left(a_{i}, g h\right)$ for $v_{h} \in \Gamma_{G}(W)_{0},\left(a_{i}, h\right) \in \Gamma_{G}(W)_{1}$ and $g \in G$. Assume $W=$ $\left(w_{1}, w_{2}, \ldots, w_{n}\right)$ is the weight sequence. Clearly, the orbit graph, $\Gamma_{G}(W) / \sim G$, is the graph with one vertex and $n$ loops. Thus, $k \Gamma_{G}(W) / \sim G$ is isomorphic to the 
free algebra in $n$ non-commuting variables via assigning to each loop a variable and then assigning each directed path its associated word in the $n$ variables, i.e.

$$
k \Gamma_{G}(W) / \sim G \cong k\left\{x_{1}, x_{2}, \ldots, x_{n}\right\} .
$$

Denote $k\left\{x_{1}, x_{2}, \ldots, x_{n}\right\}$ by $F$. It is a $G$-graded algebra by giving $x_{i}$ degree $w_{i}$ (for details, see [6]). Since the following conclusion is important, we call it Green's Theorem (see Corollary 4.5 in [6]). Note that we freely use some of the terminology from [6].

Lemma 2.3 (Green's Theorem). Let $k \Gamma_{G}(W)$ be a Hopf algebra with Hopf structure given by an allowable $k G$-bimodule structure. Let $I$ be an admissible Hopf ideal in $k \Gamma_{G}(W)$, and let $F$ be the G-graded free algebra isomorphic to $k \Gamma_{G}(W) / \sim G$, which we view as an identification. Finally let $\bar{I}$ be the ideal generated in $F$ by the orbit classes of elements of $I$. Then

(a) $\bar{I}$ is a homogeneous ideal in the free algebra $F$, and hence $F / \bar{I}$ is a finitedimensional G-graded algebra.

(b) The category of $G$-graded $F / \bar{I}$-modules (respectively, finite-dimensional $G$ graded $F / \bar{I}$-modules) is equivalent to the category of $k \Gamma_{G}(W) / I$-modules (resp. finite-dimensional $k \Gamma_{G}(W) / I$-modules $)$.

In fact, by the proof of this lemma in [6], when $I$ satisfies the conditions $G \cdot I \subset I$ and $I \cdot G \subset I$, Green's Theorem is also true. Since, clearly, $G \cdot J^{2} \subset J^{2}$ and $J^{2} \cdot G \subset J^{2}(J$ denotes the ideal generated by all arrows and see [6] for the right action of $G$ on $J$ ), we have the following corollary.

Corollary 2.4. With the notation above, the category of G-graded $F / \overline{J^{2}}$-modules (respectively, finite-dimensional G-graded $F / \overline{J^{2}}$-modules) is equivalent to the category of $k \Gamma_{G}(W) / J^{2}$-modules (resp. finite-dimensional $k \Gamma_{G}(W) / J^{2}$-modules).

\section{MAin RESUlT}

Recall that an algebra is called Nakayama if each indecomposable projective left and right module has a unique composition series. The main result of this paper is the following theorem.

Theorem 3.1. Let $H$ be a finite-dimensional basic Hopf algebra over $k$. Then $H$ is of finite representation type if and only if it is Nakayama.

The sufficiency follows immediately since it is known that every Nakayama algebra is of finite representation type ([1, p. 197). In order to prove the necessity, we need some preparations.

Lemma 3.2. Let $G$ be a group. If $k\{x, y\}$ has a $G$-graded structure and $x, y$ are homogeneous elements, then $\Lambda=k\{x, y\} /(x, y)^{2}$ has an infinite number of isoclasses of indecomposable G-graded modules.

Proof. For simplicity, we still denote the images of $x$ and $y$ in $\Lambda$ by $x$ and $y$. We denote a $G$-graded $\Lambda$-module $M$ by $M=\bigoplus_{g \in G} M_{g}$ and call $m \in M_{g}$ a homogeneous element of degree $g$. Let us construct an infinite number of isoclasses of indecomposable $G$-graded $\Lambda$-modules directly. Assume the degrees of $x$ and $y$ are $g_{x}$ and $g_{y}$ respectively for $g_{x}, g_{y} \in G$.

Let 1 be the identity element of $G$. For any natural number $n$, we construct an indecomposable $G$-graded $\Lambda$-module $Q_{n}$. As a $k$-space, $Q_{n}=k^{2 n+1}$. Let $x$ and $y$ act 
on $Q_{n}$ as the matrices $e_{n+1,1}+e_{n+2,2}+\cdots+e_{2 n, n}$ and $e_{n+2,1}+e_{n+3,2}+\cdots+e_{2 n+1, n}$ respectively where $e_{i j}$ denote the usual elementary matrices. We denote the subspace of $k^{2 n+1}$ spanned by $(0, \cdots, 0,1,0, \cdots, 0)^{T}$ by $\left(e_{i}\right)$ where the $i$-th coordinate of $(0, \cdots, 0,1,0, \cdots, 0)$ is 1 and $T$ means transpose. Then $Q_{n}$ is $G$-graded since $Q_{n}=\left(e_{1}\right)_{1} \oplus\left(e_{2}\right)_{g_{y} g_{x}^{-1}} \oplus \cdots \oplus\left(e_{n}\right)_{g_{y}^{n-1} g_{x}^{1-n}} \oplus\left(e_{n+1}\right)_{g_{x}} \oplus\left(e_{n+2}\right)_{g_{y}} \oplus\left(e_{n+3}\right)_{g_{y}^{2} g_{x}^{-1}} \oplus$

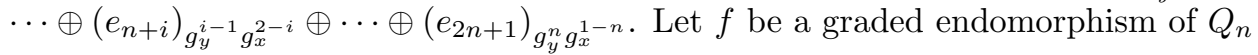
satisfying $f^{2}=f$. It is straightforward to show that $f=0$ or $f=i d$. This implies $Q_{n}$ is indecomposable as a $G$-graded $\Lambda$-module.

Corollary 3.3. Let $k\left\{x_{1}, x_{2}, \ldots, x_{n}\right\}$ be a G-graded algebra with homogeneous elements $x_{1}, x_{2}, \ldots, x_{n}$. If $n \geq 2, k\left\{x_{1}, x_{2}, \ldots, x_{n}\right\} /\left(x_{1}, x_{2}, \ldots, x_{n}\right)^{2}$ has an infinite number of isoclasses of indecomposable G-graded modules.

Proof. Note that there is a natural epimorphism as $G$-graded algebras:

$$
k\left\{x_{1}, x_{2}, \ldots, x_{n}\right\} /\left(x_{1}, x_{2}, \ldots, x_{n}\right)^{2} \stackrel{\pi}{\rightarrow} k\left\{x_{1}, x_{2}\right\} /\left(x_{1}, x_{2}\right)^{2} .
$$

Thus, through the algebra morphism $\pi$ above, we have that every $G$-graded $k\left\{x_{1}, x_{2}\right\} /\left(x_{1}, x_{2}\right)^{2}$-module is a $G$-graded $k\left\{x_{1}, x_{2}, \ldots, x_{n}\right\} /\left(x_{1}, x_{2}, \ldots, x_{n}\right)^{2}$ module. Therefore, Lemma 3.2 implies this corollary.

Proof of Theorem 3.1. We only need to prove the necessity now. Let $H$ be a basic Hopf algebra of finite representation type. By Lemma 2.2, there exist a finite group $G$ and a weight sequence $W=\left(w_{1}, w_{2}, \ldots, w_{n}\right)$ such that $H \cong k \Gamma_{G}(W) / I$ for an admissible ideal $I$. We claim $n \leq 1$. Otherwise, let $n \geq 2$. Recall that $k \Gamma_{G}(W) / \sim G \cong k\left\{x_{1}, x_{2}, \ldots, x_{n}\right\}$, which is a $G$-graded algebra by giving $x_{i}$ degree $w_{i}$. Let $I^{\prime}$ be an ideal of $k \Gamma_{G}(W)$. As before, we denote by $\overline{I^{\prime}}$ the ideal generated in $k\left\{x_{1}, x_{2}, \ldots, x_{n}\right\}$ by the orbit classes of elements of $I^{\prime}$. Let $J$ denote the ideal generated by all arrows. Then it is easy to see that $k\left\{x_{1}, x_{2}, \ldots, x_{n}\right\} / \overline{J^{2}}=$ $k\left\{x_{1}, x_{2}, \ldots, x_{n}\right\} /\left(x_{1}, x_{2}, \ldots, x_{n}\right)^{2}$. Thus by Corollary $3.3, k\left\{x_{1}, x_{2}, \ldots, x_{n}\right\} / \overline{J^{2}}$ has an infinite number of isoclasses of indecomposable $G$-graded modules. By Corollary 2.4, $k \Gamma_{G}(W) / J^{2}$ is of infinite representation type. Since $I \subset J^{2}$, there is a natural epimorphism as algebras $k \Gamma_{G}(W) / I \rightarrow k \Gamma_{G}(W) / J^{2}$. Therefore, $H \cong$ $k \Gamma_{G}(W) / I$ is of infinite representation type too. It is a contradiction. This implies $n \leq 1$.

When $n=0$, there is no arrow in $\Gamma_{G}(W)$. This means $H$ is semisimple and of course Nakayama.

When $n=1, \Gamma_{G}(W)$ is composed of a disjoint union of basic cycles (see Example 2.1). It is well known that an indecomposable elementary algebra is Nakayama if and only if its quiver is a basic cycle or a linear quiver $A_{m}$ (see [4]). Thus $H$ is Nakayama too.

Example 3.1. Let $q$ be an $n$-th primitive root of unity. Recall that the Taft algebra $T_{n^{2}}(q)$ is a Hopf algebra generated by elements $g$ and $x$, with relations

$$
g^{n}=1, \quad x^{n}=0, \quad x g=q g x
$$

with comultiplication $\Delta$, counit $\varepsilon$, and antipode $S$ given by

$$
\begin{gathered}
\Delta(g)=g \otimes g, \quad \Delta(x)=1 \otimes x+x \otimes g, \\
\varepsilon(g)=1, \quad \varepsilon(x)=0, \\
S(g)=g^{-1}, \quad S(x)=-x g^{-1} .
\end{gathered}
$$


It is a basic Hopf algebra. (This fact can be obtained from two known results, $T_{n^{2}}(q) \cong T_{n^{2}}(q)^{*}$ and $T_{n^{2}}(q)$ is a pointed Hopf algebra.) We claim that it is Nakayama. (This conclusion can also be deduced from [2].)

Denote $T_{n^{2}}(q)$ by $A$. Then $J_{A}=\operatorname{span}\left\{g^{i} x^{j} \mid i=0,1, \ldots, n-1, j=1,2, \ldots\right.$, $n-1\}$, the linear span of $\left\{g^{i} x^{j}\right\}_{0 \leq i \leq n-1,1 \leq j \leq n-1}$ and thus $J_{A}^{2}=\operatorname{span}\left\{g^{i} x^{j} \mid\right.$ $i=0,1, \ldots, n-1, j=2,3, \ldots, n-1\}$. Denote $A / J_{A}^{2}$ by $\Lambda$ and the socle of $A / J_{A}^{2}$ by $S o c \Lambda$. Then it is easy to see that

$$
\operatorname{Soc} \Lambda=J_{A} / J_{A}^{2}=\operatorname{span}\left\{g^{j} x+J_{A}^{2} \mid j=0,1, \ldots, n-1\right\} .
$$

Define a linear isomorphism

$$
f: \Lambda / J_{\Lambda} \rightarrow \operatorname{Soc} \Lambda \text { by }\left(g^{i}+J_{A}^{2}\right)+J_{\Lambda} \mapsto g^{i} x+J_{A}^{2}
$$

for $i=0,1, \ldots, n-1$. Clearly, $f$ is also a $\Lambda$-module map. Thus $\Lambda / J_{\Lambda} \cong S o c \Lambda$ as $\Lambda$-modules which implies $\Lambda$ is a self-injective algebra (see exercise 12 on p. 135 in 1]). Therefore $\Lambda$ is Nakayama by Proposition 2.16 on p. 119 in [1. It is known that an algebra $B$ is Nakayama if and only if $B / J_{B}^{2}$ is Nakayama. Since $\Lambda=A / J_{A}^{2}$, $A=T_{n^{2}}(q)$ is Nakayama. So it is of finite representation type.

Remark 3.4. In the viewpoint of representation theory, Nakayama algebras are the best understood Artin algebras next to semisimple algebras. Many properties of its representations are known. For example, we can draw its Auslander-Reiten quivers directly (see [1]). When a Nakayama algebra is elementary, its Gabriel quiver is either a basic cycle or a linear quiver $A_{m}$. Thus the Gabriel quiver and the Auslander-Reiten quiver of a basic Hopf algebra over an algebraically closed field of finite representation type are explicitly known.

\section{Classifications}

In this section we will classify all finite-dimensional pointed Hopf algebras over an algebraically closed field $k$ of finite corepresentation type. Let $H$ be a finitedimensional pointed Hopf algebra over $k$. When $H$ is cosemisimple, it is easy to see that $H \cong k G$ for a finite group $G$. So, our main task is to classify them in the non-cosemisimple case.

Recall that an algebra $A$ is called monomial if there exists a quiver $\Gamma$ and an admissible ideal $I$ generated by some paths such that $A \cong k \Gamma / I$. A coalgebra $C$ is called comonomial if $C^{*}$ is a monomial algebra. A finite-dimensional Hopf algebra is called a monomial (resp. comonomial) Hopf algebra if it is monomial (resp. comonomial) as an algebra (resp. coalgebra). So, it is obvious that a finitedimensional Hopf algebra $H$ is a monomial Hopf algebra if and only if $H^{*}$ is a comonomial Hopf algebra. One of the key observations we need in our study is the following lemma which was proved in 2] (see Corollary 2.4 in [2]).

Lemma 4.1. A non-semisimple Hopf algebra is a monomial Hopf algebra if and only if it is elementary and Nakayama.

Therefore, combining Theorem 3.1, we have the following corollary.

Corollary 4.2. Let $H$ be a finite-dimensional non-cosemisimple Hopf algebra over an algebraically closed field $k$. Then $H$ is a pointed Hopf algebra of finite corepresentation type if and only if it is a comonomial Hopf algebra. 
Proof. Equivalently, we need to prove that $H^{*}$ is a basic Hopf algebra of finite representation type if and only if it is a monomial Hopf algebra. But this is the direct consequence of Lemma 4.1 and Theorem 3.1.

So, in order to classify finite-dimensional pointed Hopf algebras over an algebraically closed field of finite corepresentation type, it is sufficient to classify comonomial Hopf algebras. In [2, the authors classified all comonomial Hopf algebras when the characteristic of $k$ is 0 (see Theorem 5.9 in [2]). Let us recall it.

Lemma 4.3. Let $k$ be an algebraically closed field with characteristic 0 . There is a one-to-one correspondence between the sets

\{the isoclasses of non-cosemisimple comonomial Hopf k-algebras\}

and

$\{$ the isoclasses of group data over $k\}$.

In the above lemma, a group data (for details, see [2]) over $k$ is defined to be a sequence $\alpha=(G, g, \chi, \mu)$ consisting of

(1) a finite group $G$, with an element $g$ in its center,

(2) a one-dimensional $k$-representation $\chi$ of $G$,

(3) an element $\mu \in k$ such that $\mu=0$ if $o(g)=o(\chi(g))$, and if $\mu \neq 0$, then $\chi^{o(\chi(g))}=1$.

Remark 4.4. For a group datum $\alpha=(G, g, \chi, \mu)$ over $k$, the corresponding comonomial Hopf algebra $A(\alpha)$ was defined in [2], which is generated as an algebra by $x$ and all $h \in G$ with relations

$$
x^{d}=\mu\left(1-g^{d}\right), \quad x h=\chi(h) h x, \quad \forall h \in G
$$

where $d=o(\chi(g))$. Its comultiplication $\Delta$, counit $\varepsilon$, and antipode $S$ are defined by

$$
\begin{gathered}
\Delta(x)=g \otimes x+x \otimes 1, \quad \varepsilon(x)=0, \\
\Delta(h)=h \otimes h, \quad \varepsilon(h)=1 \quad \forall h \in G, \\
S(x)=-g^{-1} x, \quad S(h)=h^{-1}, \quad \forall h \in G .
\end{gathered}
$$

For any quiver $\Gamma$, we define $C_{d}(\Gamma):=\bigoplus_{i=1}^{d-1} k \Gamma(i)$ for $d \geq 2$, where $\Gamma(i)$ is the set of all paths of length $i$ in $\Gamma$. We denote the basic cycle of length $n$ (Example $2.1)$ by $Z_{n}$ and denote $C_{d}\left(Z_{n}\right)$ by $C_{d}(n)$. In [7, we get the following conclusion (see Theorem 4.2 in [7]).

Lemma 4.5. Let $H$ be a non-cosemisimple comonomial Hopf algebra over $k$ of characteristic $p$. Then there exist a $d_{0}$-th primitive root of unity $q \in k$ with $d_{0} \mid n$, $r \geq 0$ and $d=p^{r} d_{0} \geq 2$ such that

$$
H \cong C_{d}(n) \oplus \cdots \oplus C_{d}(n)
$$

as coalgebras and

$$
H \cong C_{d}(n) \#_{\sigma} k(G / N)
$$

as Hopf algebras, where $G=G(H)$, the set of group-like elements of $H$, and $N=$ $G\left(C_{d}(n)\right)$, the set of group-like elements of $C_{d}(n)$.

Summarizing the above conclusions, we have the following classification theorem of finite-dimensional pointed Hopf algebras of finite corepresentation type. 
Theorem 4.6. (A) Let $H$ be a finite-dimensional pointed Hopf algebra of finite corepresentation type over an algebraically closed field $k$. Then

(A.1) if $H$ is cosemisimple, then $H \cong k G$ for some finite group $G$;

(A.2) if $H$ is not cosemisimple and the characteristic of $k$ is zero, then $H \cong A(\alpha)$ for some group datum $\alpha=(G, g, \chi, \mu)$ where $A(\alpha)$ was defined in the above remark;

(A.3) if $H$ is not cosemisimple and the characteristic of $k$ is $p$, then there exists a $d_{0}$-th primitive root of unity $q \in k$ with $d_{0} \mid n, r \geq 0$ and $d=p^{r} d_{0} \geq 2$ such that

$$
H \cong C_{d}(n) \oplus \cdots \oplus C_{d}(n)
$$

as coalgebras and

$$
H \cong C_{d}(n) \#_{\sigma} k(G / N)
$$

as Hopf algebras, where $G=G(H)$ and $N=G\left(C_{d}(n)\right)$.

(B) Let $H$ be a finite-dimensional Hopf algebra. If

(B.1) $H \cong k G$ for some finite group $G$ or

(B.2) $H \cong A(\alpha)$ for some group datum $\alpha=(G, g, \chi, \mu)$ where $A(\alpha)$ was defined in the above remark or

(B.3) $H \cong C_{d}(n) \oplus \cdots \oplus C_{d}(n)$ as coalgebras, then $H$ is a pointed Hopf algebra of finite corepresentation type.

Proof. (A.1) is explained in the first paragraph of this section. By using Lemma 4.3 and Lemma 4.5, (A.2) and (A.3) can be obtained directly as long as we note that $H$ is a comonomial Hopf algebra now.

Since $k G$ is cosemisimple and clearly pointed, (B.1) implies $H$ is a pointed Hopf algebra of finite corepresentation type.

By Lemma 4.3 and Remark 4.4, $A(\alpha)$ is a comonomial Hopf algerba and thus (B.2) implies $H$ is a comonomial Hopf algebra. Therefore, by Corollary $4.2, H$ is a pointed Hopf algebra of finite corepresentation type.

It is known that $C_{d}(n)$ is a comonomial coalgebra (see [2]). From this fact we know that (B.3) implies $H$ is a comonomial Hopf algebra. Thus, by Corollary 4.2 again, $H$ is a pointed Hopf algebra of finite corepresentation type.

Remark 4.7. (1) In order to not cause confusion, we introduced the concept of comonomial Hopf algebras. Note that, in [2] and [7], comonomial Hopf algebra in this paper was called monomial Hopf algebra.

(2) Part (A) of Theorem 4.6 gives the structures of finite-dimensional pointed Hopf algebras of finite corepresentation type. In some sense, Part (B) of Theorem 4.6 is the converse of Part (A) since Part (B) implies that the structures given in Part (A) are precisely all finite-dimensional pointed Hopf algebras of finite corepresentation type.

\section{ACKNOWLEDGMENT}

The authors are grateful to the referee for his/her important and valuable comments.

\section{REFERENCES}

[1] M. Auslander, I. Reiten, and S. Smalø, Representation theory of Artin algebras, Cambridge University Press, 1995. MR 1314422 (96c:16015)

[2] X-W. Chen, H-L. Huang, Y. Ye, and P. Zhang, Monomial Hopf algebras, J. Algebra 275 (2004), 212-232. MR2047446 (2005e:16060) 
[3] C. Cibils, and M. Rosso, Hopf quivers, J. Algebra 254(2) (2002), 241-251. MR1933868 (2003h:16016)

[4] Yu.A. Drozd, and V.V. Kirichenko, Finite dimensional algebras, Springer-Verlag, BerlinHeidelberg-New York-Tokyo, 1993. MR1284468 (95i:16001)

[5] E. Green, Graphs with relations, coverings and group-graded algebras, Trans. Amer. Math. Soc. 279 (1983), 297-310. MR.0704617 (85a:16033)

[6] E. Green, and Ø. Solberg, Basic Hopf algebras and quantum groups, Math.Z 229 (1998), 45-76. MR 1649318(2000h:16049)

[7] G-X. Liu, and Y. Ye, Monomial Hopf algebras over fields of positive characteristic, Sci. China Ser. A, in press (available at http://www.cms.zju.edu.cn/index.asp?ColumnName=pdfbook).

Institute of Mathematics, Academy of Mathematics and Systems Science, Chinese Academy of Sciences, Beijing 100080, People's Republic of China

E-mail address: gxliu@amss.ac.cn

Department of Mathematics, Zhejiang University, Hangzhou, Zhejiang 310028, PeoPLE'S Republic OF China

E-mail address: fangli@zju.edu.cn 